\title{
Comparative genomics of Alternaria species provides insights into the pathogenic lifestyle of Alternaria brassicae - a pathogen of the Brassicaceae family
}

\author{
Sivasubramanian Rajarammohan ${ }^{1,2}$, Kumar Paritosh $^{3}$, Deepak Pental ${ }^{3}$ and Jagreet Kaur ${ }^{1 *}$ (D)
}

\begin{abstract}
Background: Alternaria brassicae, a necrotrophic pathogen, causes Alternaria Leaf Spot, one of the economically important diseases of Brassica crops. Many other Alternaria spp. such as A. brassicicola and A. alternata are known to cause secondary infections in the A. brassicae-infected Brassicas. The genome architecture, pathogenicity factors, and determinants of host-specificity of A. brassicae are unknown. In this study, we annotated and characterised the recently announced genome assembly of A. brassicae and compared it with other Alternaria spp. to gain insights into its pathogenic lifestyle.
\end{abstract}

Results: We also sequenced the genomes of two A. alternata isolates that were co-infecting B. juncea using Nanopore MinION sequencing for additional comparative analyses within the Alternaria genus. Genome alignments within the Alternaria spp. revealed high levels of synteny between most chromosomes with some intrachromosomal rearrangements. We show for the first time that the genome of $A$. brassicae, a large-spored Alternaria species, contains a dispensable chromosome. We identified $460 \mathrm{~A}$. brassicae-specific genes, which included many secreted proteins and effectors. Furthermore, we have identified the gene clusters responsible for the production of Destruxin-B, a known pathogenicity factor of $A$. brassicae.

Conclusion: The study provides a perspective into the unique and shared repertoire of genes within the Alternaria genus and identifies genes that could be contributing to the pathogenic lifestyle of A. brassicae.

Keywords: Alternaria spp., Comparative genomics, Destruxin B, Dispensable chromosome, Necrotroph

\section{Background}

The genus Alternaria belonging to the class of Dothideomycetes contains many important plant pathogens. Diseases in the Brassicaceae family caused by Alternaria spp. result in significant yield losses [1]. Alternaria spp. have a wide host range within the Brassicaceae, infecting both the vegetable as well as the oilseed crops. Some of the most damaging species include Alternaria brassicae, A. brassicicola, A. alternata, A. raphani, A. japonicus, and A. tenuissima. A. brassicae preferentially infects the oleiferous Brassicas while the others are more devastating

\footnotetext{
* Correspondence: jagreet@south.du.ac.in

${ }^{1}$ Department of Genetics, University of Delhi , South Campus, New Delhi

110021, India

Full list of author information is available at the end of the article
}

on the vegetable Brassicas. A. brassicae is particularly more damaging in the hilly regions of the Indian subcontinent, where conducive climatic conditions allow it to profusely reproduce and cause infections on almost all parts of the plant. Extensive screening for resistance to $A$. brassicae in the cultivated Brassica germplasms has not revealed any source of resistance [2].

The factors that contribute to the pathogenicity of $A$. brassicae are relatively unknown. Pathogenicity of many Alternaria spp. has been mainly attributed to the secretion of host-specific toxins (HSTs). HSTs induce pathogenesis on a rather narrow species range and are mostly indispensable for pathogenicity. At least $12 \mathrm{~A}$. alternata pathotypes have been reported to produce HSTs and thereby cause disease on different species [3]. Many of 
the HST producing genes/gene clusters have been found on supernumerary chromosomes or dispensable chromosomes [4]. A. brassicae has been reported to produce low molecular weight cyclic depsipeptides named destruxins. Destruxin B is known to be a major phytotoxin and is reported to be a probable HST of A. brassicae [5, 6]. Additionally, a proteinaceous HST (ABR-toxin), was isolated from the spore germination fluid of $A$. brassicae but was only partially characterised [7].

Genome sequencing and comparative analysis can help identify shared and species-specific pathogenicity factors in closely-related species. Genomic information for nearly 26 Alternaria spp. including A. brassicae is currently available and has contributed immensely to clarify the taxonomy of the Alternaria genus [8]. However, comparative analyses to identify pathogenicity factors that confer the ability to infect a wide range of hosts have not been carried out. Most of the genomic information available for Alternaria spp. has been generated by shotgun sequencing approaches and hence is fragmented. A contiguous genome assembly is essential, especially when the aim is to identify and characterise pathogenicity factors or effectors, which are often present in rapidly evolving repeat-rich regions of the genome [9]. Additionally, contiguous genome assemblies enable an accurate prediction of genes and gene clusters that are involved in various secondary metabolic processes, many of which are implicated to have an important role in pathogenicity. Long reads generated from Pacific Biosciences (PacBio) single-molecule real-time (SMRT) sequencing technology and Oxford Nanopore sequencing technology enable the generation of high-quality genome assemblies at affordable costs. Besides the recently announced nearcomplete genome sequence of $A$. brassicae [10], three other near-complete genomes of Alternaria spp. have been reported recently [11-13].

Alternaria Leaf spot in the field usually occurs as a mixed infection of $A$. brassicae and other Alternaria species, such as $A$. brassicicola and A. alternata. It is however not known whether the $A$. alternata infecting Brassicas represent a separate pathotype with a different range of host-specific toxin(s) or are just facultative pathogens. We, therefore, carried out Nanopore-based sequencing of two A. alternata isolates that were recovered from an A. brassicae-infected B. juncea plant.

Given the invasiveness of $A$. brassicae and the lack of information on its pathogenicity factors, we undertook the current study to 1) functionally annotate and characterise the recently announced genome of $A$. brassicae, 2) sequence and analyse the genomes of two A. alternata isolates co-infecting $B$. juncea with respect to the genome of $A$. alternata isolated from very divergent hosts, 3) analyse the repertoire of CAZymes, secondary metabolite encoding gene clusters, and effectors in $A$. brassicae, and 4) carry out a comparative analysis of the genomes sequenced in this study with some of the previously sequenced Alternaria spp. genomes to gain insights into their pathogenic lifestyles.

\section{Results and discussion}

Genomic features of $A$. brassicae and two other coinfecting $A$. alternata isolates

We sequenced the genomes of two isolates of $A$. alternata (PN1 and PN2) that were co-infecting $B$. juncea with $A$. brassicae. The A. brassicae assembly has been previously described [10]. Briefly, the assembly consisted of nine complete chromosomes and one chromosome with telomeric repeats missing at one of the ends. Apart from these chromosomes, there were six contigs of which one of them was $\sim 1 \mathrm{Mb}$ in size, which may together constitute a dispensable chromosome (Fig. 1). The N50 of the A. brassicae assembly was $2.98 \mathrm{Mb}$ (Table 1). The two isolates co-infecting $B$. juncea were identified to be $A$. alternata based on their ITS and GAPDH sequences. The A. alternata assemblies Aat PN1 and Aat_PN2 consisted of 14 contigs totalling to $33.77 \mathrm{Mb}$, and 15 contigs totalling to $33.53 \mathrm{Mb}$, respectively (Table 1). Six contigs in each of the two assemblies contained telomeric repeats on both ends and therefore, are most likely to represent full chromosomal molecules. Four other contigs in both the assemblies contained telomeric repeats on one end but were of similar size of full chromosome molecules as described in A. solani [13]. Therefore, the genome assemblies for A. alternata isolates represented ten nearly-complete chromosomes of each of the two isolates.

Whole genome alignments with related Alternaria spp. showed an overall synteny between the genomes with minor rearrangements (Fig. 2). Additionally, mitochondrial sequences were also obtained from the sequencing data for the two isolates of A. alternata. The mitochondrial genomes of the A. alternata strains were approximately $49,783 \mathrm{bp}$ and $50,765 \mathrm{bp}$ in size respectively and showed high similarity with the previously published mitochondrial genome of $A$. alternata [14].

Gene prediction following repeat masking resulted in the identification of 11593, 11495, and 11387 genes in the A. brassicae, A. alternata PN1, and PN2 genome assemblies, respectively. This was comparable to the gene numbers estimated in other Alternaria spp. (Table 1). BUSCO analysis showed that the gene models predicted in the three genomes covered $98 \%$ of the single copy conserved fungal genes indicating near-completeness of the assemblies. The predicted genes were comprehensively annotated using a combination of databases as described in the Methods section (Fig. 1). In addition to the three genomes, we also predicted genes de novo in the genome assemblies of three other Alternaria species 




Fig. 1 Summary of A. brassicae genome, (From outer to inner circular tracks) a pseudochromosomes/scaffolds, b Protein-coding genes, c Repeat elements, $\mathbf{d}$ Transposable Elements (DNA and LTR), e predicted secondary metabolite clusters, $\mathbf{f}$ Secreted proteins, $\mathbf{g}$ predicted effectors

which were sequenced using long-read technologies viz. A. brassicicola (abra43), A. alternata (ATCC34957), and A. solani (altNL03003) (Table 1). These six genomes and their gene predictions were used for the comparative analyses of secondary metabolite encoding gene clusters and effector-coding genes.

Phylogenomic analysis assigns a separate clade for the Brassica-infecting $A$. brassicae and $A$. brassicicola within the Alternaria genus

In order to accurately reconstruct the divergence and relationship between $A$. brassicae, the two A. alternata isolates (PN1 and PN2), and the other Alternaria species, we conducted phylogenomic analyses using 29 single copy orthologs that had the highest phylogenetic signal as calculated by the program Mirlo. Selection of genes with higher phylogenetic signals leads to phylogenies that are more congruent with the species tree [15]. The resulting phylogeny showed that the large-spored Alternaria and small-spored Alternaria species clustered separately into two different clades (Fig. 3). Interestingly, the two major pathogens of the Brassicas viz. A. brassicae and $A$. brassicicola clustered separately from all the other Alternaria species, possibly indicating a different

Table 1 Assembly statistics of the six near-complete Alternaria genome sequences

\begin{tabular}{|c|c|c|c|c|c|c|}
\hline & A. brassicae $\mathrm{J}^{\mathrm{a}}$ & A. alternata PN1 & A. alternata PN2 & A. solani altNL03003 ${ }^{b}$ & A. brassicicola abra43 ${ }^{c}$ & A. alternata ATCC $34957^{d}$ \\
\hline Assembly size (Mb) & 34.14 & 33.77 & 33.53 & 32.78 & 31.04 & 33.48 \\
\hline No. of contigs & 17 & 14 & 15 & 10 & 29 & 27 \\
\hline $\begin{array}{l}\text { No. of contigs } \\
(>10,000 \mathrm{bp})\end{array}$ & 17 & 13 & 15 & 10 & 29 & 25 \\
\hline Largest contig (Mb) & 7.1 & 6.86 & 6.76 & 6.94 & 3.3 & 3.96 \\
\hline N50 & 2.98 & 3.09 & 3.1 & 2.87 & 2.1 & 2.83 \\
\hline GC (\%) & 50.7 & 50.98 & 50.95 & 51.32 & 50.85 & 50.95 \\
\hline Repeat content (\%) & 9.33 & 2.43 & 2.64 & 5.71 & 9.3 & 2.71 \\
\hline Predicted genes & 11,593 & 11,495 & 11,387 & 11,804 & 10,261 & 12,500 \\
\hline
\end{tabular}

a - [10], b - [13], c - [11], d - [12] 


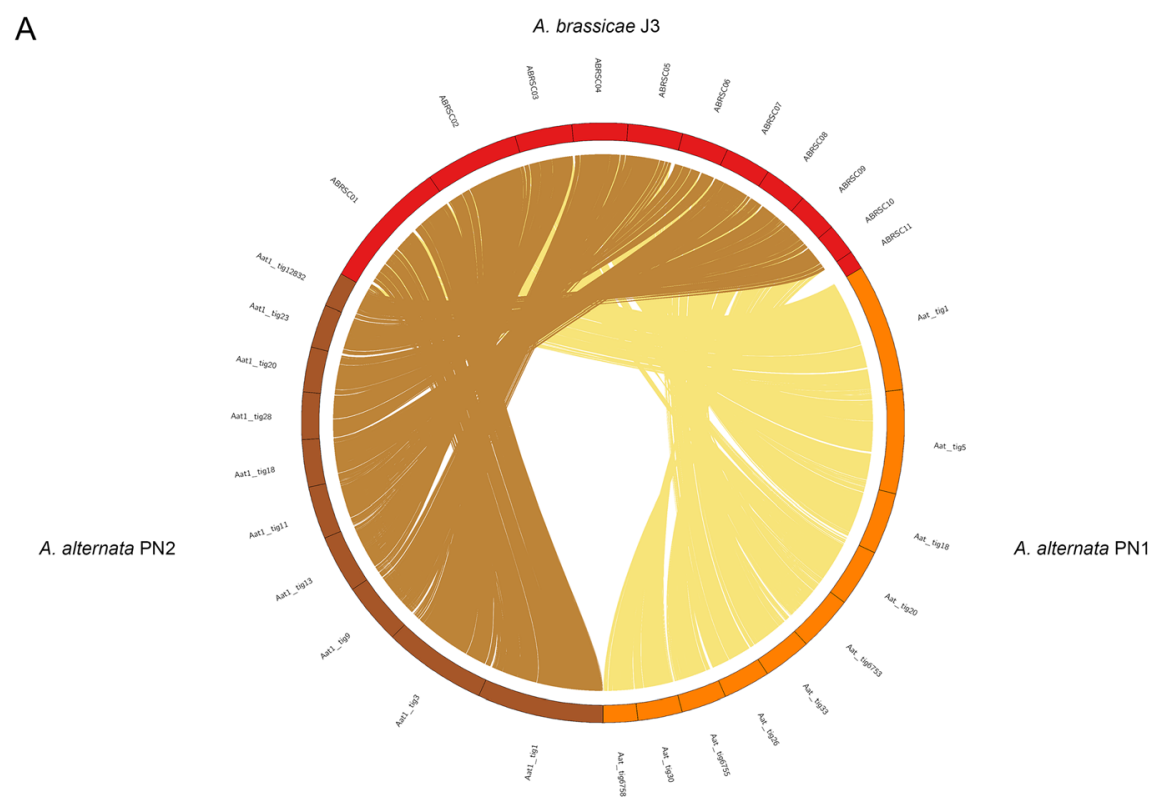

B

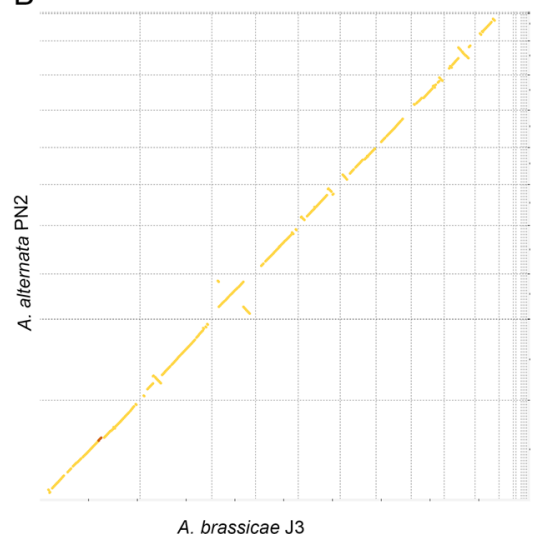

C

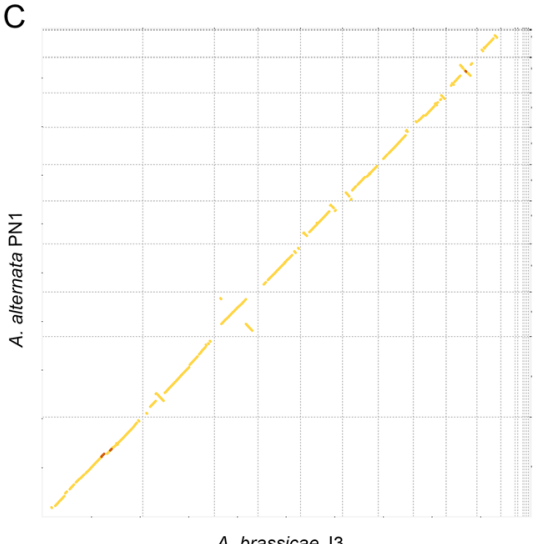

Fig. 2 Whole-genome alignments of A. alternata PN1 and PN2 with A. brassicae. a Circos plot showing macrosynteny of A. alternata PN1 and PN2 with $A$. brassicae across all contigs except the dispensable contigs (ABRSC11, scaffold13,17,18,19), b and $\mathbf{c}$ Syntenic dotplots of $A$. brassicae with $A$. alternata PN1 and PN2

evolutionary trajectory based on the common host preferences of these two species.

\section{Comparative analyses of $A$. alternata isolates obtained from different hosts}

We compared the genomes of $A$. alternata PN1 and PN2 (isolated from $B$. juncea) to that of $A$. alternata ATCC34957 (isolated from sorghum) to identify any differences in their genomic content that might allow these to infect two very different species. Whole-genome alignments of $A$. alternata $\mathrm{PN} 1$ and $\mathrm{PN} 2$ to that of $A$. alternata ATCC34957 revealed very high levels of synteny and the absence of any species-specific regions. We identified 719, 152, and 586 isolate-specific genes between the three isolates of $A$. alternata, respectively (Additional file 1: Table S1). More than two-third of the isolate-specific genes in all the three isolates were uncharacterized proteins or had no annotations. Notably, all the three isolates did not contain any dispensable chromosomes which may confer pathogenicity, as has been reported for $A$. alternata isolates infecting many of the fruit crops such as citrus, pear, and apple [16-18]. The gene repertoires of the three isolates also consisted of similar number and type of effectors, CAZymes, and secondary metabolite clusters (Table 2). Additionally, the two isolates PN1 and PN2 do not cause infection symptoms on their own in B. juncea under epiphytotic conditions (data not shown). Our results suggest that these isolates of $A$. alternata (PN1 and PN2) may be facultative pathogens that lead a saprophytic lifestyle and may change over to a pathogenic lifestyle under certain environmental conditions. 


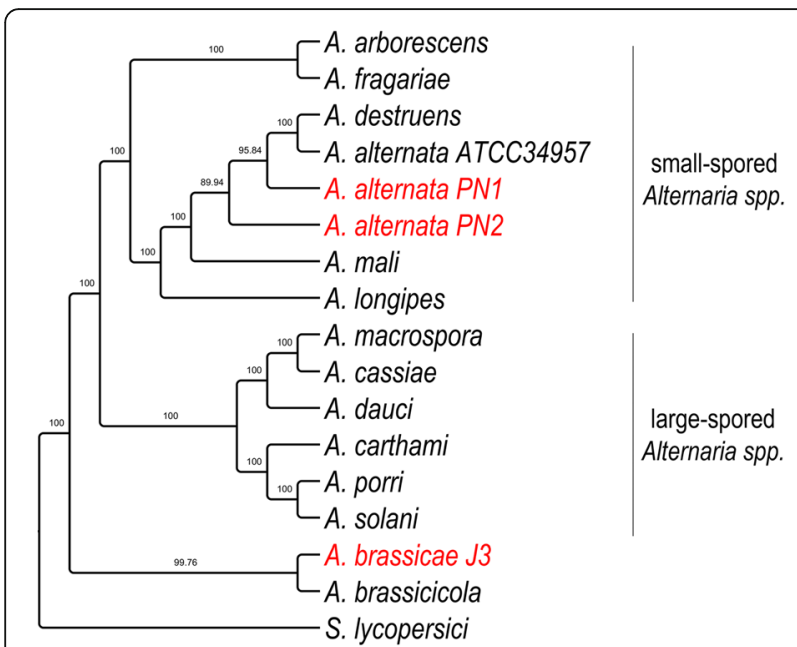

Fig. 3 Phylogenetic tree of Alternaria species with S. lycopersici as an outgroup. The tree was constructed using 29 single copy orthologs, which had the highest phylogenetic signal as calculated in Mirlo. Branch support values from 1000 bootstrap replicates are shown

\section{An abundance of repeat-rich regions and transposable elements in $A$. brassicae}

Filamentous plant pathogens tend to have a distinct genome architecture with higher repeat content. Repeat content estimation and masking using RepeatModeler and RepeatMasker revealed that the $A$. brassicae genome consisted of $\sim 9.33 \%$ repeats as compared to 2.43 and $2.64 \%$ repeats in the $A$. alternata genomes. The A. brassicae genome harbors the highest repeat content $(\sim 9.33 \%)$ among all the Alternaria species sequenced till date. Our analysis showed that the repeat content differs significantly between the $A$. alternata isolates and the other pathogenic Alternaria species. The pathogenic Alternaria species especially $A$. brassicae and $A$. brassicicola had a considerably larger repertoire of LTR/Gypsy and LTR/Copia elements (> 8X) in comparison to the other A. alternata isolates (pathogenic and non-pathogenic) (Fig. 4). The $A$. brassicae and $A$. brassicicola genomes also had an overrepresentation of DNA transposons, which amounted to 5\% of the genome, as compared to $<1 \%$ in the other Alternaria species (Fig. 4).

This proliferation of repetitive DNA and subsequent evolution of genes overlapping these regions may be the key to evolutionary success wherein these pathogens have managed to persist over generations of co-evolutionary conflict with their hosts. Proximity to TEs potentially exposes the genes to Repeat-Induced Point Mutations (RIP) and therefore accelerated evolution [19, 20]. Ectopic recombination between similar TEs may also result in new combinations of genes and thereby increase the diversity of proteins or metabolites.

\section{Presence of a dispensable chromosome in the large- spored $A$. brassicae}

Lineage-specific (LS) chromosomes or dispensable chromosomes (DC) have been reported from several phytopathogenic species including $A$. alternata. DCs in $A$. alternata are known to confer virulence and hostspecificity to the isolate. The whole-genome alignments of $A$. brassicae with other Alternaria spp. revealed that a contig of approx. $1 \mathrm{Mb}$ along with other smaller contigs (66-366 kb) was specific to $A$. brassicae and did not show synteny to any region in the other Alternaria spp. However, partial synteny was observed when the contig was aligned to the sequences of other dispensable chromosomes reported in Alternaria spp. [16, 17]. This led us to hypothesize that these contigs together may represent a DC of $A$. brassicae. To confirm this, we searched the contigs for the presence of AaMSAS and ALT1genes, which are known marker genes for dispensable chromosomes in Alternaria spp. [4]. We found two copies of the AaMSAS gene as part of two secondary metabolite biosynthetic clusters on the $1 \mathrm{Mb}$ contig. However, we did not find any homolog of the ALT1 gene. Additionally, the repeat content of the contigs (ABRSC11, scaffold 13, 17, 18, and 19) was compared to the whole genome. The gene content of the lineage-specific contigs was significantly lower than that of the core chromosomes (Table 3). Conversely, the DC contigs were highly enriched in TE content as compared to the core chromosomes (Table 3).

Although, the DC was not enriched with genes encoding secreted proteins, the proportion of secreted effector genes was $30 \%$ higher as compared to the core chromosomes. All the above evidence point to the fact that A. brassicae may indeed harbour a DC. DCs in Alternaria spp. have been reported so far from only the

Table 2 Protein repertoires and functional classification of the six near-complete Alternaria genome sequences

\begin{tabular}{|c|c|c|c|c|c|c|}
\hline & A. brassicae J3 & A. alternata PN1 & A. alternata PN2 & A. brassicicola abra43 & A. solani altNL03003 & A. alternata ATCC34957 \\
\hline Total proteins & 11,593 & 11,495 & 11,387 & 10,261 & 11,804 & 12,500 \\
\hline CAZymes & 508 & 542 & 550 & 484 & 538 & 571 \\
\hline Peptidases & 277 & 259 & 253 & 262 & 283 & 299 \\
\hline Secreted proteins & 1195 & 1284 & 1243 & 1052 & 1358 & 1414 \\
\hline Effectors & 198 & 219 & 212 & 160 & 227 & 252 \\
\hline
\end{tabular}



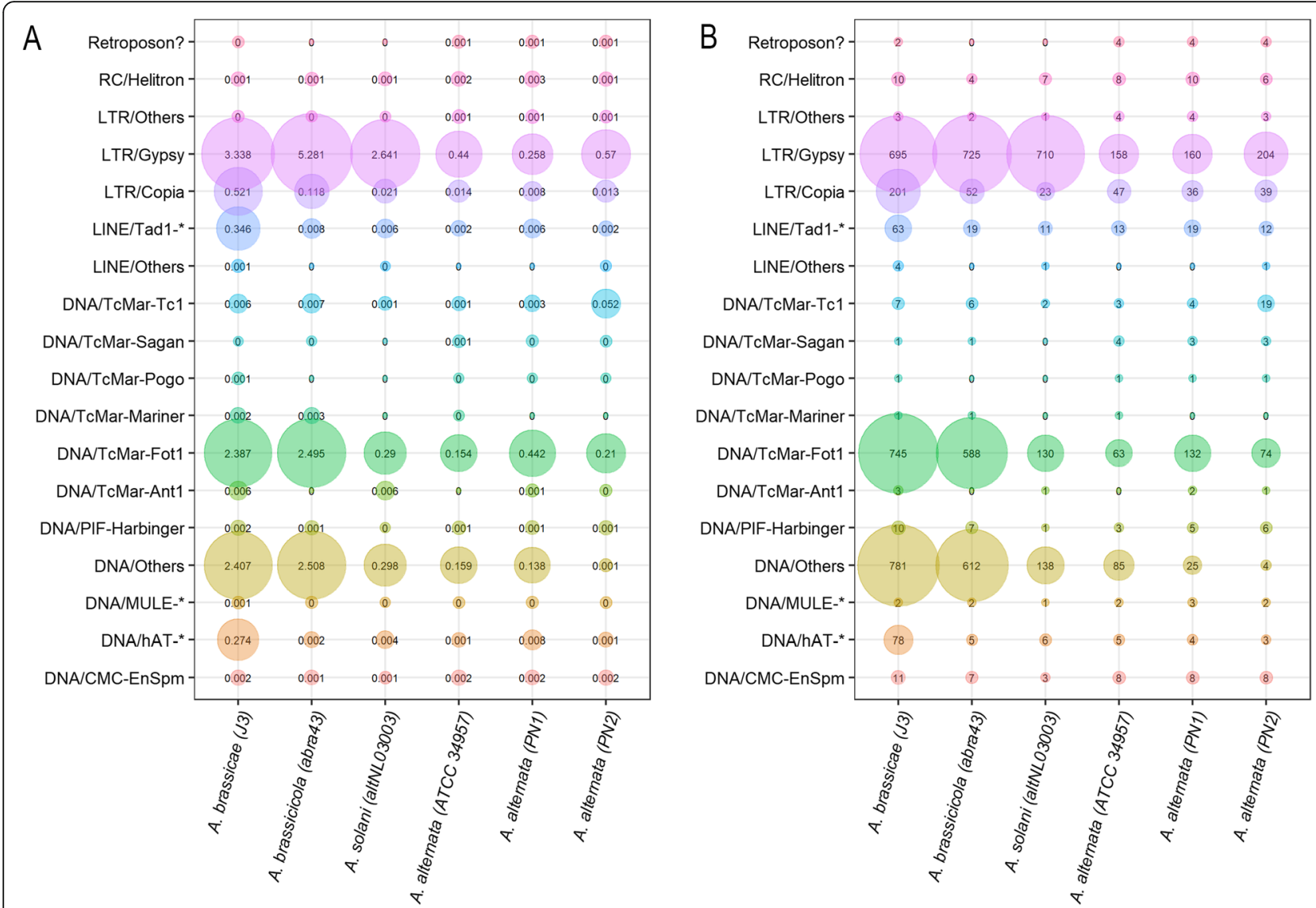

Fig. 4 Comparison of repeat content in six Alternaria species. The size of the bubbles corresponds to the (a) percentage of transposable elements (TES) in the genome, $\mathbf{b}$ copy number of the TE in the genome

small-spored Alternaria spp. and no large-spored Alternaria species have been known to harbour DCs. It remains to be seen whether the DC contributes to virulence of $A$. brassicae. Future studies would involve the characterization of the dispensable chromosome in A. brassicae and correlating its presence to the pathogenicity of different isolates.

Table 3 Comparison of characteristics of Core chromosomes and dispensable chromosome of A. brassicae

\begin{tabular}{lll}
\hline Characteristic & Core chromosomes & DC contigs (all) \\
\hline Total length (bp) & $32,140,555$ & $1,809,659$ \\
$\mathrm{G}+\mathrm{C}(\%)$ & 50.85 & 47 \\
Number of protein-coding genes & 11,216 & 377 \\
$\begin{array}{l}\text { Proportion of genes by length (\%) } \\
\text { Number of Transposable element }\end{array}$ & 52.48 & 30.05 \\
$\begin{array}{l}\text { (TE) copies } \\
\text { Proportion of TEs by length (\%) }\end{array}$ & 5.78 & 313 \\
$\begin{array}{l}\text { Proportion of secreted protein } \\
\text { genes (\%) }\end{array}$ & 10.09 & 20.89 \\
Proportion of effector genes (\%) & 1.69 & 9.81 \\
\hline
\end{tabular}

Orthology analysis reveals species-specific genes with putative roles in virulence

Differences in gene content and diversity within genes contribute to adaptation, growth, and pathogenicity. In order to catalogue the differences in the gene content within the Alternaria genus and the Dothideomycetes, we carried out an orthology analysis on the combined set of 3,60,216 proteins from 30 different species (including 16 Alternaria species) belonging to Dothideomycetes (Additional file 2: Table S2) out of which 3,45,321 proteins could be assigned to atleast one of the orthogroups. We identified 460 A. brassicae specific genes which were present in $A$. brassicae but absent in all other Alternaria species (Additional file 3: Table S3). These species-specific genes included 35 secreted protein coding genes out of which 11 were predicted to be effectors. Additionally, 20 of these species-specific genes were present on the DC. A large number of these proteins belonged to the category of uncharacterised proteins with no known function. In order to test whether these species-specific genes are the result of adaptive evolution taking place in the repeat-rich regions of the 
genome, we carried out a permutation test to compare the overlap of repeat-rich regions and transposable elements with a random gene set against the overlap of these species-specific genes. We found that these species-specific genes overlapped significantly with repeat-rich regions $(P$ value: 9.99e-05; Z-score: - 4.825) and transposable elements (P-value: 0.0460; Z-score: 2.539 ) in the genome.

\section{Secondary metabolite profile of $A$. brassicae and its association with transposable elements (TEs)}

The genera of Alternaria and Cochliobolus are known to be the major producers of host-specific secondary metabolite toxins. Alternaria spp. especially are known for the production of chemically diverse secondary metabolites, which include the host-specific toxins (HSTs) and nonHSTs. These secondary metabolites are usually generated by non-ribosomal peptide synthases (NRPS) and polyketide synthases (PKS). We identified five NRPS type SM gene clusters, 12 PKS type gene clusters and seven terpene-like gene clusters in A. brassicae (Additional file 4: Table S4). Out of the five NRPS clusters, we could identify three clusters which produce known secondary metabolites viz. Destruxin B, HC-toxin and dimethylcoprogen (siderophore) with known roles in virulence.

The gene cluster responsible for dimethylcoprogen (siderophore) production in A. brassicae consists of 22 genes, including the major biosynthetic genes, oxidoreductases, and siderophore transporters. Siderophores are iron-chelating compounds, used by fungi to acquire extracellular ferric iron and have been reported to be involved in fungal virulence [21]. The identification of the gene cluster responsible for siderophore synthesis would enable the study of siderophores and their role in pathogenicity in A. brassicae. Additionally, a PKS type cluster consisting of 12 genes, responsible for melanin production was also identified (Additional file 4: Table S4). The melanin biosynthetic cluster has been described for $A$. alternata previously [22]. Also, the transcription factor Amr1, which induces melanin production, has been characterized in A. brassicicola and is known to suppress virulence [23]. However, the role of melanin in virulence is ambiguous and species-specific [24-26].

The plant pathogens belonging to the genus of Alternaria seem to have a dynamic capacity to acquire new secondary metabolite potential to colonize new ecological niches. The most parsimonious explanation for this dynamic acquisition of secondary metabolite potential is horizontal gene transfer within the genus of Alternaria and possibly with other genera. There is extensive evidence in the literature that much of the HSTs of Alternaria are carried on the dispensable chromosomes and exchange of these chromosomes can broaden the host specificity $[4,18,27]$. We also identified an NRPS cluster, possibly coding for $\mathrm{HC}$-toxin in one of the DCs (scaffold 18) (Additional file 4: Table S4). HC-toxin is a known virulence determinant of the plant pathogen Cochliobolus carbonum, which infects maize genotypes that lack a functional copy of HM1, a carbonyl reductase that detoxifies the toxin [28]. A recent report showed that $A$. jesenskae also could produce HC-toxin, making it the only other fungus other than $C$. carbonum to produce the toxin [29]. The presence of HC-toxin gene cluster, a virulence determinant in C. carbonum, in a DC of $A$. brassicae points to the fact that interspecies horizontal gene transfer may be more common than expected.

Apart from horizontal gene transfer, rapid duplication, divergence and loss of the SM genes may also contribute to the pathogen evolving new metabolic capabilities. These processes of duplication and divergence may well be aided by the proximity of the secondary metabolite clusters to the repeat elements that makes them prone to RIP-mutations. Therefore, we tested whether the secondary metabolite clusters were also associated with repeat-rich regions. A permutation test was used to compare the overlap of repeat-rich regions with a random gene set against the overlap of secondary metabolite cluster genes. The secondary metabolite clusters significantly overlapped repeat-rich regions as compared to the random gene set ( $P$-value: 0.0017; Z-score: 2.7963). Also, these clusters overlapped significantly with transposable elements among the repeat-rich regions $(P$ value: 0.0087 ; $Z$-score: 2.9871 ). This shows that both the mechanisms described above for the acquisition of new secondary metabolite potential may be possible in the case of $A$. brassicae. Population-scale analyses at the species and genus level may throw light on the prevalence of these mechanisms within the Alternaria genus.

\section{Synteny analysis reveals the genetic basis of the exclusivity of Destruxin B production by $A$. brassicae within the Alternaria genus}

Destruxin B represents a class of cyclic depsipeptides that is known to be one of the key pathogenicity factors of $A$. brassicae and has been reported to be a hostspecific toxin of $A$. brassicae [5]. Destruxin B has not been reported to be produced by any of the other Alternaria species. Here we report for the first time the biosynthetic gene clusters responsible for Destruxin $\mathrm{B}$ production in $A$. brassicae. The cluster consists of 10 genes, including the major biosynthetic enzyme encoded by an NRPS gene (DtxS1) and the rate-limiting enzyme, DtxS3 (aldo-keto reductase) (Additional file 4: Table S4). Interestingly, synteny analysis of this cluster among the six Alternaria species showed that both these genes were not present in any of the other Alternaria spp. although the overall synteny of the cluster was maintained in all of these species (Fig. 5). The absence of the key genes coding for the enzymes DtxS1 and DtxS3 in the 


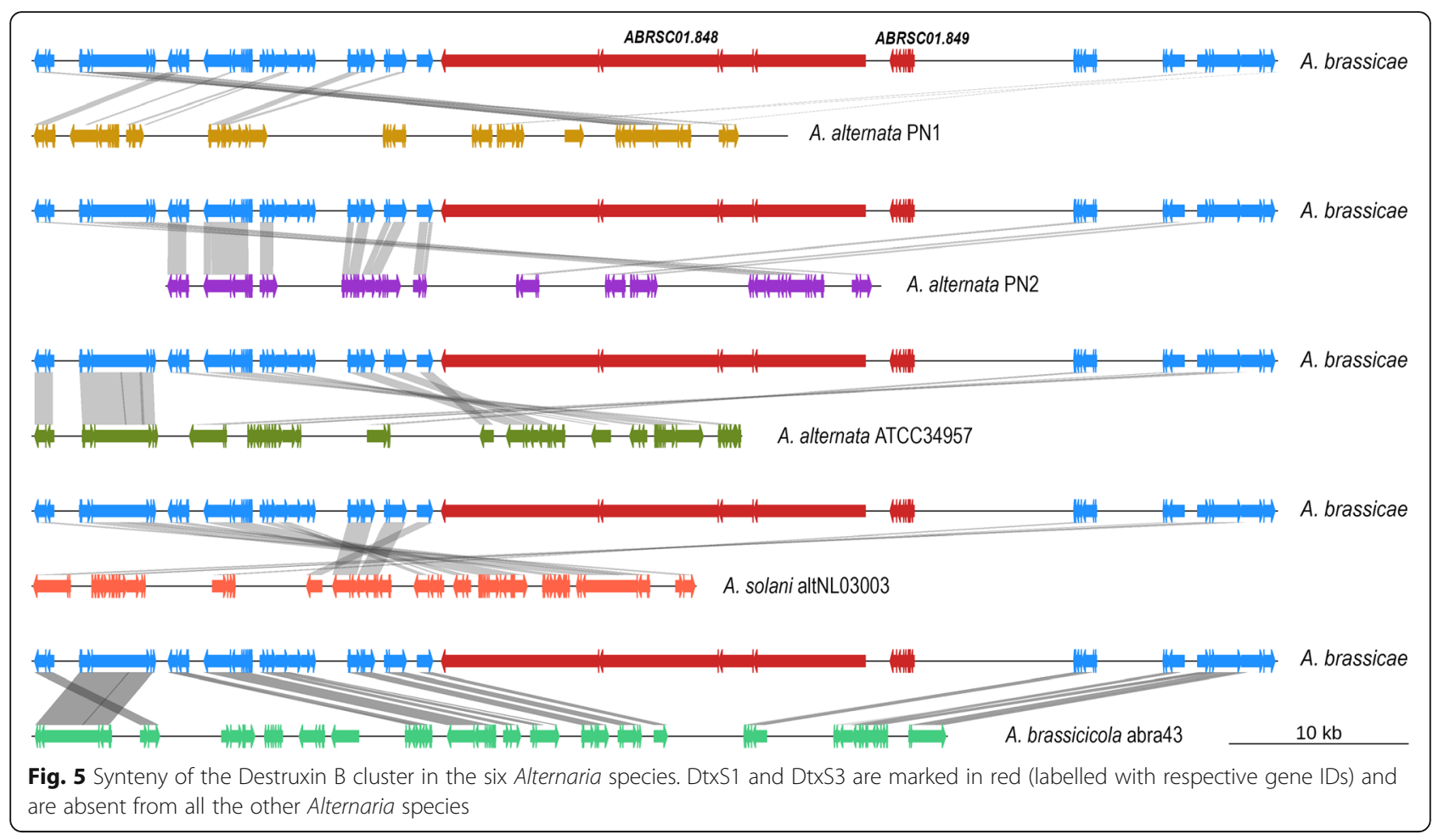

Destruxin B cluster in the other Alternaria species explains the absence of Destruxin B in those species.

Destruxin B has been reported from the entomopathogen Metarhizium robertsii [30], and Ophiosphaerella herpotricha [27], the cause of spring dead spot of bermudagrass, apart from A. brassicae. Wang et al., described the secondary metabolite biosynthetic cluster responsible for Destruxin production in $M$. robertsii [31]. The Destruxin cluster in the Metarhizhium species consists of two additional key genes viz. DtxS2 and DtxS4. DtxS2 is responsible for the conversion of Destruxin B into Destruxin A, C, D, and $\mathrm{E}$. We could not find any syntenic gene in the cluster that corresponds to DtxS2 or DtxS4 in A. brassicae. A. brassicae is known to produce only Destruxin $B$ and not the other forms of Destruxin, which might possibly explain the absence of DtxS2 in A. brassicae. DtxS4 is an aspartate decarboxylase which is required for the production of $\beta$ alanine from aspartic acid and is essential for the production of the backbone structure of Destruxin. Moreover, deletion mutants of DtxS4 were able to produce Destruxin when they were supplemented with $\beta$-alanine in the medium [31]. Even though DtxS4 is not present in the Destruxin cluster, A. brassicae has one functional copy of aspartate decarboxylase present elsewhere in the genome and therefore can possibly ensure the supply of $\beta$-alanine for the synthesis of Destruxin B. The evolutionary history of Destruxin B production within the Metarhizhium genus closely resembled that of Alternaria. The specialist pathogens such as $M$. acridum lack the two key enzymes and hence do not produce Destruxins. However, $M$. robertsii, a generalist with a wider host range produces Destruxins [31]. It was therefore hypothesized that Destruxins might be responsible for the establishment of the extended host range of $M$. roberstii. A similar hypothesis may also be true in the case of $A$. brassicae, which has a broad host range and can infect almost all the Brassicaceae. Further experiments to determine the host range of Destruxin null mutants of $A$. brassicae may help clarify the role of this important phytotoxin in extending the host range of $A$. brassicae.

\section{Distinct CAZyme profiles of $A$. brassicae and $A$. brassicicola within the Alternaria genus}

CAZymes (Carbohydrate-Active enZymes) are proteins involved in the degradation, rearrangement, or synthesis of glycosidic bonds. Plant pathogens secrete a diverse range of CAZymes that breakdown the complex polysaccharides in the plant cell wall. They consist of five distinct classes viz. Glycoside hydrolases (GH), Glycosyltransferases (GT), Polysaccharide lyases (PL), Carbohydrate esterases (CE), and Carbohydrate-binding modules (CBM). We identified $>500$ CAZymes in the six Alternaria spp. including A. brassicae (Additional file 5: Table S5). The CAZyme distribution of $A$. brassicae and A. brassicicola varied from those of the other Alternaria spp. thus forming a separate cluster (Fig. 6). The number of auxiliary activity enzymes or the enzymes involved in plant cell wall degradation varied considerably between the different 

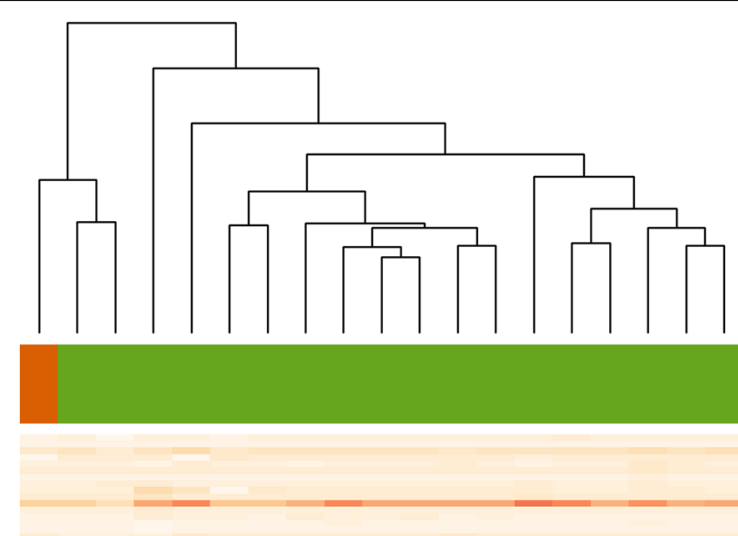

\section{Auxiliary Activity -AA \\ (ligninases and LPMOs)}

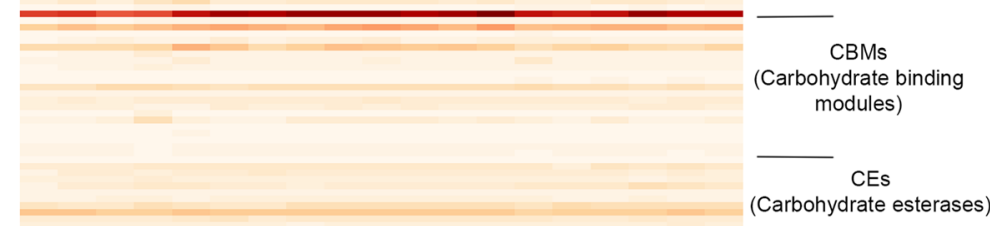

GHs

(Glycoside hydrolases)

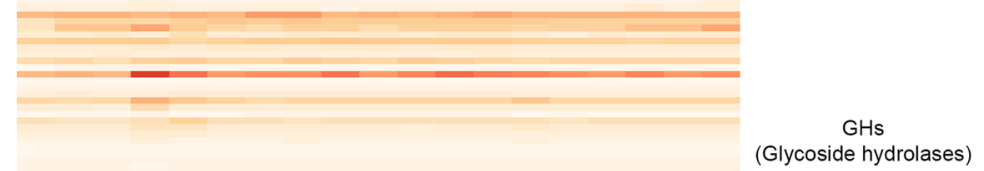

GTs

(Glycosyl transferases)
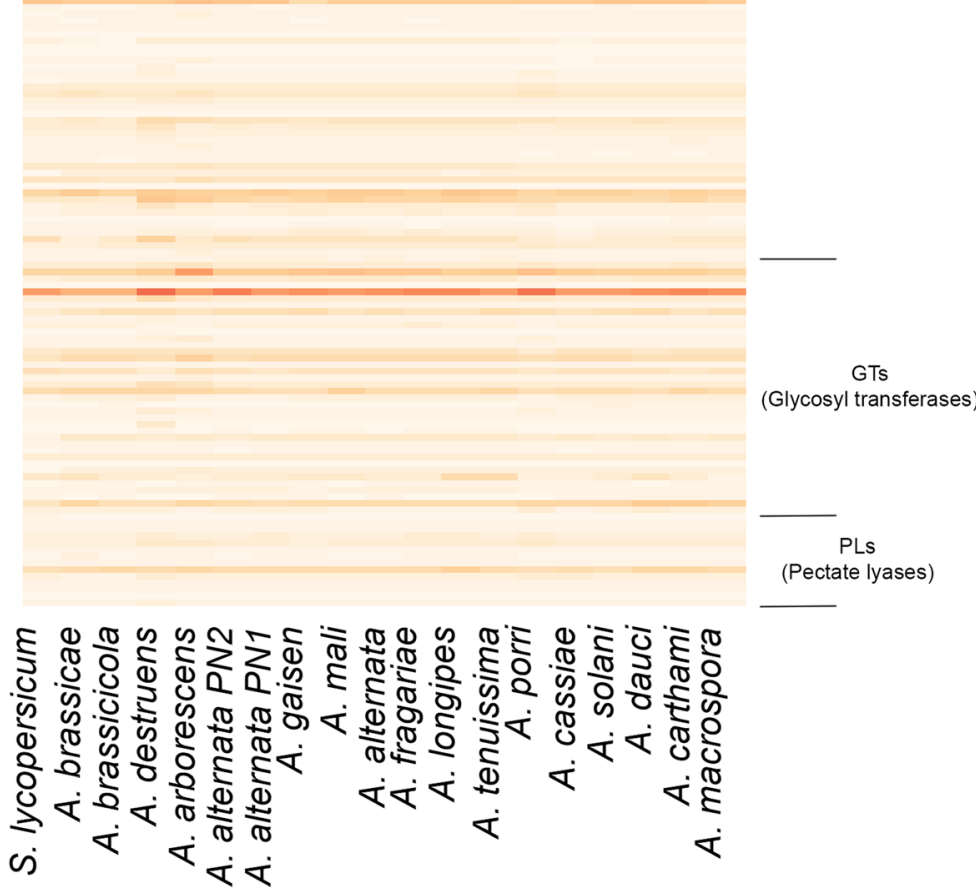

Fig. 6 Heatmap of the CAZyme profiles in 16 species with Alternaria genus. The clustering of the organisms closely resembles that of the species phylogeny except A. brassicae and A. brassicicola, which cluster separately with the outgroup Stemphylium lycopersici. The CAZymes were grouped according to the CAZy classification 
species compared. Nearly $46 \%$ of the CAZymes in A. brassicae were secreted out of which $\sim 17 \%$ were predicted to be effectors.

The AA9 family (formerly GH61; copper-dependent lytic polysaccharide monooxygenases (LPMO)) is significantly enlarged in comparison to the other CAZy families in the Alternaria genus with each species containing $>20$ copies of the gene. The copy numbers in the Alternaria spp. are much higher than the copy numbers reported for Botrytis and Fusarium spp. [32]. The AA9 family is involved in the degradation of cell-wall polysaccharides and are known to act on a range of polysaccharides including starch, xyloglucan, cellodextrins, and glucomannan. LPMOs have been hypothesized to have a dual role - directly cleaving the cell -wall polysaccharides, and acting as a ROS generator and thus contributing to the oxidative stress leading to necrosis in the plant tissues [33, 34]. Strikingly, 11 of the 26 AA9 proteins present in A. brassicae are predicted to be secreted effectors. Characterisation of these CAZymes and their role in pathogenesis could be the subject of further studies.

\section{Overlapping effector profiles within the Alternaria genus}

We predicted the effector repertoire of six Alternaria species (Table 2) including $A$. brassicae using the pipeline described in the methods section. Most of the small secreted proteins predicted to be effectors were uncharacterised/predicted proteins and thus may be novel species or genus-specific effectors. A. brassicae had the largest proportion of unknown/predicted/hypothetical proteins in the set of candidate effectors. We found that most of the effectors between the six species to be common and overlapping, suggesting that the broad mechanisms of pathogenesis involving proteinaceous effectors may be conserved within the genus. However, we found two copies of a beta/gamma-crystallin fold containing protein to be present only in A. brassicae and A. brassicicola and not in the other four Alternaria species. A further search through the nr database of NCBI revealed that this protein was completely absent in the Alternaria genus and the closest matches were found in other plant pathogens viz. Macrophomina and Fusarium species. However, no information is available as to its function or role in pathogenicity in any of the species.

We could also establish that some of the effectors in $A$. brassicae have the potential to evolve adaptively since they were also significantly associated with the repeatrich regions of the genome ( $P$-value: 0.0003 ; Z-score: 2.8778). Population-level analyses are therefore required to identify the effectors under positive selection, which could shed light on the evolution of pathogenicity in $A$. brassicae. The effectors identified in this study reveal the wide range of proteins that may be involved in the pathogenesis of A. brassicae. 39 of these effectors were predicted to be CAZymes having various roles in the degradation of the cell wall and associated polysaccharides. The genome of $A$. brassicae contained two necrosis and ethylene-inducing peptide proteins, which have been implicated in the pathogenesis of various pathogens including oomycetes and necrotrophs [35-37]. Hrip1 (Hypersensitive response inducing protein 1) from $A$. alternata has recently been shown to be recognized by MdNLR16 in a classical gene-for-gene manner, and deletion of Hrip1 from A. alternata enhances its virulence [38]. A Hrip1 homolog is also present in A. brassicae, but it is not predicted to be secreted outside the cell, although this needs to be verified experimentally. The presence of effectors which are recognized in a gene-forgene manner opens up the possibility of identification of complementary R-genes in the host that can be utilized for developing resistant varieties or cultivars.

\section{Conclusions}

A. brassicae has an enormous economic impact on the cultivated Brassica species worldwide, particularly the oleiferous types. Using the recently published highquality genome assembly of $A$. brassicae, we annotated the genome and carried out comparative analyses of $A$. brassicae with other Alternaria spp. to discern unique features of A. brassicae vis-à-vis the other Alternaria species. We sequenced and annotated the genomes of two $A$. alternata isolates that were co-infecting $B$. juncea. The two A. alternata isolates had a gene content, effector repertoire, and CAZyme profiles that were very similar to that of an earlier sequenced A. alternata isolate (ATCC34957). This leads us to conclude that these isolates are opportunistic pathogens with a limited ability to cause infection on their own but would contribute overall to the disease outcome of a primary A. brassicae infection. Additionally, we show the presence of a dispensable chromosome in A. brassicae, a large-spored Alternaria species for the first time. The implications of a lineage-specific dispensable chromosome in $\mathrm{A}$. brassicae towards pathogenesis remains to be unravelled. We also described the CAZyme profiles of nearly 30 Doth $i$ deomycetes and show that the CAZyme profiles of $A$. brassicae and $A$. brassicicola are different from the other Alternaria species. We also identified several important secondary metabolite gene clusters with putative roles in pathogenicity. The identification of the biosynthetic cluster responsible for Destruxin B in A. brassicae paves the way for reverse genetics studies to conclusively determine the contribution of Destruxin B towards the pathogenicity of $A$. brassicae. The repertoire of effectors identified in the six Alternaria species was largely overlapping. It may thus be hypothesised that host-specificity in the Alternaria species may be conferred by the 
combined action of proteinaceous effectors and the secondary metabolite toxins. Future studies would involve characterisation of the effectors and secondary metabolite clusters identified in this study and elucidating their role in pathogenesis.

\section{Methods}

\section{Genome sequencing and assembly}

Two isolates of $A$. alternata which were found to be coinfecting $B$. juncea leaves along with $A$. brassicae in our experimental field station at Delhi, India (PN1 and PN2) were isolated and purified by single spore culture. High molecular-weight genomic DNA was extracted from mycelia of 5-day old cultures of $A$. alternata isolates using a method described earlier [10]. $2 \mu \mathrm{g}$ of the high molecularweight genomic DNA was used for Nanopore library preparation using LSK-108 ONT ligation protocol. The libraries were then run on R9.4 SpotON MinION flowcells for $24 \mathrm{~h}$. Live base calling was enabled for all the runs. The MinION runs produced 4,14,210 and 2,68,910 reads amounting to 2.36 GB and 1.98 GB of data for A. alternata PN1 and PN2, respectively. The genomes were assembled de novo using the Canu assembler (version 1.6) [39]. Nanopolish (version 0.7.1) was used to compute an improved consensus sequence using the signal-level raw data for the assemblies.

\section{mRNA sequencing and transcript reconstruction}

Total RNA was isolated from 15-day old fungal mycelia of $A$. brassicae, grown on Potato Dextrose Agar (PDA) and Radish Root Sucrose Agar (RRSA), using Qiagen RNeasy Plant Mini Kit following the manufacturer's instructions. $100 \mathrm{bp}$ sequencing libraries were prepared using the TruSeq Stranded mRNA Library Prep Kit (Illumina). Paired-end sequencing was carried out using Illumina SBS v4 chemistry on an Illumina Hiseq 2500. The Hiseq run generated 50 million PE reads per sample. The raw reads were trimmed using Trimmomatic, and the trimmed reads were then mapped to the de novo genome assembly using STAR (version 2.5.0) [40]. Transcripts were reconstructed using Cufflinks (version 2.2.1) [41] and likely coding regions were identified using TransDecoder (version 5.4.0) [42].

\section{Gene prediction and annotation}

RepeatModeler (version 1.0.11) was used for de novo repeat family identification. The de novo identified repeat library was used for masking the genome using RepeatMasker (version 4.0.7). The repeat-masked genome was used for gene predictions. For gene prediction, multiple lines of gene evidence were integrated using EVM (EvidenceModeler version 1.1.1) [43]. Two ab initio gene callers were used viz. AUGUSTUS (version 3.3.1) [44] and GeneMark-HMM-ES (version 2.0) [45]. GeneMark-HMM-ES was self-trained on the repeat-masked genome whereas AUGUSTUS was trained on the genome and cDNA hints from A. alternata. RNA-Seq evidence in the form of coding regions identified by TransDecoder was also used. Additionally, homologybased gene prediction was carried out using GeMoMa (version 1.4.2) [46] with protein-coding genes of $A$. longipes, $A$. arborescens and $A$. alternata.

Each of these lines of evidence was presented to EVM as separate tracks. In EVM, weights were assigned to each evidence as follows: AUGUSTUS 1, GeneMarkHMM-ES 1, GeMoMa 1, and RNA-Seq evidence 5. The genes predicted by EVM were used for all the downstream analyses. Genes were then annotated using BLAST (version 2.7.1+) against UniProt, SWISS-PROT, CAZy, MEROPS, and PHI-BASE. The fungal version of antiSMASH (version 4.0) [47] was used to identify secondary metabolite gene clusters in the genomes.

Candidate effector proteins were predicted using the following pipeline: a) SignalP (version 4.1) [48] and Phobius to identify secreted proteins, b) TMHMM (version 2.0) [49] to remove proteins with transmembrane domains, c) predGPI to filter out proteins that harbored a GPI membrane-anchoring domain, and d) EffectorP (version 2.0) [50] to predict potential effectors from the abovefiltered protein set.

\section{Orthology and whole-genome phylogenetic analysis}

The genomes of 16 Alternaria species (Additional file 2: Table S2) were included in the analysis with Stemphylium lycopersici as an outgroup. The proteomes of the fungi were clustered using the Orthofinder (version 2.2.6) [51] pipeline with default parameters. The clusters were further analysed with Mirlo (https:/github.com/ mthon/mirlo) to identify phylogenetically informative single copy gene families. These families were then concatenated into one large alignment and used for phylogenetic analysis. A phylogenetic tree was then inferred from the concatenated alignment by the neighbour joining method and Jukes Cantor substitution model using 1000 replicates. Orthologs were also identified for 13 other pathogenic Dothideomycetes (Additional file 2: Table S2) for comparative analyses using the same pipeline as above.

\section{Relationship of TEs and repeat-rich regions to genes and gene clusters}

The overlap of repeat-rich regions and transposable elements (TEs) with i) genes encoding secreted proteins, ii) effectors and iii) secondary metabolite gene clusters were analysed using the regioneR package [52] in R. The analysis involved comparison of the overlap of each the above gene sets with transposable elements and repeatrich regions with a random set of genes selected from the genome. A distribution of means was established by running 10,000 permutation tests, which was then used to calculate a $p$-value. 


\section{Supplementary information}

Supplementary information accompanies this paper at https://doi.org/10. 1186/s12864-019-6414-6.

Additional file 1: Table S1. Isolate-specific genes between the three isolates of A. alternata viz. AatPN1, AatPN2, and ATCC34957.

Additional file 2: Table S2. List of Dothideomycetes used in the orthology analysis.

Additional file 3: Table S3. List of 460 A. brassicae species-specific proteins with gene coordinates and description.

Additional file 4: Table S4. List of predicted secondary metabolite gene clusters in A. brassicae along with their constituent genes, coordinates in the genome, and their description.

Additional file 5: Table S5. Comparison of the CAZyme profiles of the 30 Dothideomycetes including 16 Alternaria species.

\section{Abbreviations}

CBM: Carbohydrate-binding modules; CE: Carbohydrate esterases; DC: Dispensable chromosome; GH: Glycoside hydrolases; GT: Glycosyltransferases; HST: Host-specific toxin; LPMO: Lytic polysaccharide monooxygenases; NRPS: Non-ribosomal peptide synthase; PKS: Polyketide synthase; PL: Polysaccharide lyases; RIP: Repeat-induced Point mutation; ROS: Reactive oxygen species; SMRT: Single-molecule real-time; TE: Transposable elements

\section{Acknowledgements}

The abstract and part of the data was presented as a poster during the 2019 XVIII International Society for Molecular Plant-microbe interactions (IS-MPMI) Congress and the abstract was published as a supplement in the MPMI journal (https://apsjournals.apsnet.org/doi/pdfplus/10.1094/MPMl-32-10-S1.1).

\section{Authors' contributions}

$J K, D P$, and SR made contributions to conception and design of the major objectives of the study. KP contributed to sequencing of the A. alternata isolates and helped with the comparative analyses. SR carried out the sequencing, did the comparative analyses and wrote the first draft of the manuscript. JK, DP, and SR revised the manuscript. All authors read and approved the final version of the manuscript.

\section{Funding}

This work was supported by the grants from the Department of Biotechnology (DBT), Government of India [BT/IN/Indo-UK/CGAT/12/DP/ 2014-15 and BT/01/NDDB/UDSC/2016]. The funding bodies played no role in the design of the study and collection, analysis, and interpretation of data and in writing the manuscript.

\section{Availability of data and materials}

The genome assembly and associated raw data generated in this study have been deposited under the National Center for Biotechnology Information BioProject PRJNA526360, PRJNA548052, and PRJNA548054. Additional data files and related scripts are available at https://github.com/siva5/Alternariacomparative-genomics.git.

\section{Ethics approval and consent to participate}

Not applicable.

\section{Consent for publication}

Not applicable.

\section{Competing interests}

The authors declare that they have no competing interests.

\section{Author details}

'Department of Genetics, University of Delhi , South Campus, New Delhi 110021, India. ${ }^{2}$ Present Address: National Agri-Food Biotechnology Institute, Mohali, India. ${ }^{3}$ Centre for Genetic Manipulation of Crop Plants, University of Delhi South Campus, New Delhi, India.
Received: 9 August 2019 Accepted: 19 December 2019

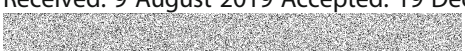

\section{References}

1. Nowicki M, Nowakowska M, Niezgoda A, Kozik E. Alternaria Black Spot of Crucifers: Symptoms, Importance of Disease, and Perspectives of Resistance Breeding. Vege Crops Res Bullet. 2012;76(1):5.

2. Sharma G, Dinesh Kumar V, Haque A, Bhat SR, Prakash S, Chopra VL. Brassica coenospecies: a rich reservoir for genetic resistance to leaf spot caused by Alternaria brassicae. Euphytica. 2002;125(3):411-7.

3. Nishimura S, Kohmoto K. Host-specific toxins and chemical structures from Alternaria species. Annu Rev Phytopathol. 1983;21:87-116.

4. Akagi $Y$, Akamatsu $H$, Otani $H$, Kodama M. Horizontal chromosome transfer, a mechanism for the evolution and differentiation of a plant-pathogenic fungus. Eukaryot Cell. 2009:8(11):1732-8.

5. Bains PS, Tewari JP. Purification, chemical characterization and hostspecificity of the toxin produced by Alternaria brassicae. Physiol Mol Plant Pathol. 1987;30(2):259-71.

6. BUCHWALDT L, GREEN H. Phytotoxicity of destruxin B and its possible role in the pathogenesis of Alternaria brassicae. Plant Pathol. 1992;41(1):55-63.

7. Parada RY, Sakuno E, Mori N, Oka K, Egusa M, Kodama M, Otani H. Alternaria brassicae produces a host-specific protein toxin from germinating spores on host leaves. Phytopathology. 2008;98(4):458-63.

8. Woudenberg JH, Groenewald JZ, Binder M, Crous PW. Alternaria redefined. Stud Mycol. 2013;75(1):171-212.

9. Thomma B, Seidl MF, Shi-Kunne X, Cook DE, Bolton MD, van Kan JAL, Faino L. Mind the gap; seven reasons to close fragmented genome assemblies. Fungal Genet Biol. 2016;90:24-30.

10. Rajarammohan S, Pental D, Kaur J. Near-complete genome assembly of Alternaria brassicae - a necrotrophic pathogen of Brassica crops. Mol PlantMicrobe Interact. 2019;(32)28:928-30.

11. Belmas E, Briand M, Kwasiborski A, Colou J, N'Guyen G, lacomi B, Grappin P, Campion C, Simoneau P, Barret M, et al. Genome Sequence of the Necrotrophic Plant Pathogen Alternaria brassicicola Abra43. Genome Announc. 2018;6(6).

12. Nguyen HD, Lewis $C T$, Levesque CA, Grafenhan T. Draft Genome Sequence of Alternaria alternata ATCC 34957. Genome Announc. 2016:4(1):e01554-15.

13. Wolters PJ, Faino L, van den Bosch TBM, Evenhuis B, Visser RGF, Seidl MF, Vleeshouwers $V$. Gapless genome assembly of the potato and tomato early blight pathogen Alternaria solani. Mol Plant-Microbe Interact. 2018;31(7): 692-4.

14. Liao M, Chen C, Li Q. The complete mitochondrial genome of Alternaria alternata (Hypocreales: Nectriaceae). Mitochondrial DNA Part B. 2017;2(2):587-8.

15. Salichos $L$, Rokas A. Inferring ancient divergences requires genes with strong phylogenetic signals. Nature. 2013;497(7449):327-31.

16. Hu J, Chen C, Peever T, Dang H, Lawrence C, Mitchell T. Genomic characterization of the conditionally dispensable chromosome in Alternaria arborescens provides evidence for horizontal gene transfer. BMC Genomics. 2012;13:171.

17. Armitage AD, Cockerton HM, Sreenivasaprasad S, Woodhall J, Lane C, Harrison RJ, Clarkson JP. Genomics, evolutionary history and diagnostics of the Alternaria alternata species group including apple and Asian pear pathotypes. bioRxiv. 2019:534685.

18. Johnson LJ, Johnson RD, Akamatsu H, Salamiah A, Otani H, Kohmoto K, Kodama M. Spontaneous loss of a conditionally dispensable chromosome from the Alternaria alternata apple pathotype leads to loss of toxin production and pathogenicity. Curr Genet. 2001;40(1):65-72.

19. Galagan JE, Selker EU. RIP: the evolutionary cost of genome defense. Trends Genet. 2004;20(9):417-23.

20. John Clutterbuck A. Genomic evidence of repeat-induced point mutation (RIP) in filamentous ascomycetes. Fungal Genet Biol. 2011;48(3):306-26.

21. Oide S, Moeder W, Krasnoff S, Gibson D, Haas H, Yoshioka K, Turgeon BG. NPS6, encoding a nonribosomal peptide synthetase involved in siderophoremediated iron metabolism, is a conserved virulence determinant of plant pathogenic ascomycetes. Plant Cell. 2006;18(10):2836-53.

22. Kimura N, Tsuge T. Gene cluster involved in melanin biosynthesis of the filamentous fungus Alternaria alternata. J Bacteriol. 1993;175(14):4427-35.

23. Cho Y, Srivastava A, Ohm RA, Lawrence CB, Wang KH, Grigoriev IV, Marahatta SP. Transcription factor Amr1 induces melanin biosynthesis and suppresses virulence in Alternaria brassicicola. PLoS Pathog. 2012:8(10): e1002974. 
24. Howard RJ, Valent B. Breaking and entering: host penetration by the fungal rice blast pathogen Magnaporthe grisea. Annu Rev Microbiol. 1996;50:491-512.

25. Fry W, Yoder O, Apple A. Influence of naturally occurring marker genes on the ability of Cochliobolus heterostrophus to induce field epidemics of southern corn leaf blight. Phytopathology. 1984;74(12):175-8.

26. TANABE K, PARK P, TSUGE T, KOHMOTO K, NISHIMURA S. Characterization of the mutants of Alternaria alternata Japanese pear pathotype deficient in melanin production and their pathogenicity. Ann Phytopathol Soc Jpn. 1995;61(1):27-33.

27. Venkatasubbaiah P, Tisserat NA, Chilton WS. Metabolites of Ophiosphaerella herpotricha, a cause of spring dead spot of bermudagrass. Mycopathologia. 1994;128(3):155-9.

28. Walton JD. HC-toxin. Phytochemistry. 2006;67(14):1406-13.

29. Wight WD, Labuda R, Walton JD. Conservation of the genes for HC-toxin biosynthesis in Alternaria jesenskae. BMC Microbiol. 2013;13:165.

30. Giuliano Garisto Donzelli B, Krasnoff SB, Moon YS, Churchill AC, Gibson DM. Genetic basis of destruxin production in the entomopathogen Metarhizium robertsii. Curr Genet. 2012;58(2):105-16.

31. Wang B, Kang Q, Lu Y, Bai L, Wang C. Unveiling the biosynthetic puzzle of destruxins in Metarhizium species. Proc Natl Acad Sci U S A. 2012;109(4): 1287-92.

32. Johansen KS. Lytic polysaccharide Monooxygenases: the microbial power tool for lignocellulose degradation. Trends Plant Sci. 2016;21(11):926-36.

33. Amselem J, Cuomo CA, van Kan JA, Viaud M, Benito EP, Couloux A, Coutinho PM, de Vries RP, Dyer PS, Fillinger S, et al. Genomic analysis of the necrotrophic fungal pathogens Sclerotinia sclerotiorum and Botrytis cinerea. PLoS Genet. 2011;7(8):e1002230.

34. Simon UK, Polanschutz LM, Koffler BE, Zechmann B. High resolution imaging of temporal and spatial changes of subcellular ascorbate, glutathione and $\mathrm{H}(2) \mathrm{O}(2)$ distribution during Botrytis cinerea infection in Arabidopsis. PLoS One. 2013;8(6):e65811.

35. Bae H, Kim MS, Sicher RC, Bae HJ, Bailey BA. Necrosis- and ethyleneinducing peptide from Fusarium oxysporum induces a complex cascade of transcripts associated with signal transduction and cell death in Arabidopsis. Plant Physiol. 2006;141(3):1056-67.

36. Oome S, Van den Ackerveken G. Comparative and functional analysis of the widely occurring family of Nep1-like proteins. Mol Plant-Microbe Interact. 2014;27(10):1081-94

37. Qutob D, Kemmerling B, Brunner F, Kufner I, Engelhardt S, Gust AA, Luberacki B, Seitz HU, Stahl D, Rauhut T, et al. Phytotoxicity and innate immune responses induced by Nep1-like proteins. Plant Cell. 2006;18(12): 3721-44.

38. Meng D, Li C, Park HJ, Gonzalez J, Wang J, Dandekar AM, Turgeon BG, Cheng $\mathrm{L}$. Sorbitol modulates resistance to Alternaria alternata by regulating the expression of an NLR resistance gene in Apple. Plant Cell. 2018;30(7): 1562-81.

39. Koren S, Walenz BP, Berlin K, Miller JR, Bergman NH, Phillippy AM. Canu: scalable and accurate long-read assembly via adaptive k-mer weighting and repeat separation. Genome Res. 2017;27(5):722-36.

40. Dobin A, Gingeras TR. Mapping RNA-seq Reads with STAR. Curr Protoc Bioinformatics. 2015:51:11 14 11-9.

41. Trapnell C, Roberts A, Goff L, Pertea G, Kim D, Kelley DR, Pimentel H, Salzberg SL, Rinn JL, Pachter L. Differential gene and transcript expression analysis of RNA-seq experiments with TopHat and cufflinks. Nat Protoc. 2012;7(3):562-78.

42. Haas BJ, Papanicolaou A, Yassour M, Grabherr M, Blood PD, Bowden J, Couger MB, Eccles D, Li B, Lieber M, et al. De novo transcript sequence reconstruction from RNA-seq using the trinity platform for reference generation and analysis. Nat Protoc. 2013;8(8):1494-512.

43. Haas BJ, Salzberg SL, Zhu W, Pertea M, Allen JE, Orvis J, White O, Buell CR, Wortman JR. Automated eukaryotic gene structure annotation using EVidenceModeler and the program to assemble spliced alignments. Genome Biol. 2008;9(1):R7.

44. Stanke M, Schoffmann O, Morgenstern B, Waack S. Gene prediction in eukaryotes with a generalized hidden Markov model that uses hints from external sources. BMC Bioinformatics. 2006;7:62.

45. Ter-Hovhannisyan V, Lomsadze A, Chernoff YO, Borodovsky M. Gene prediction in novel fungal genomes using an ab initio algorithm with unsupervised training. Genome Res. 2008;18(12):1979-90.
46. Keilwagen J, Wenk M, Erickson JL, Schattat MH, Grau J, Hartung F. Using intron position conservation for homology-based gene prediction. Nucleic Acids Res. 2016;44(9):e89.

47. Blin K, Wolf T, Chevrette MG, Lu X, Schwalen CJ, Kautsar SA, Suarez Duran HG, de Los Santos ELC, Kim HU, Nave M, et al. antiSMASH 4.0-improvements in chemistry prediction and gene cluster boundary identification. Nucleic Acids Res. 2017;45(W1):W36-41.

48. Petersen TN, Brunak S, von Heijne G, Nielsen H. SignalP 4.0: discriminating signal peptides from transmembrane regions. Nat Methods. 2011;8(10):785-6.

49. Krogh A, Larsson B, von Heijne G, Sonnhammer EL. Predicting transmembrane protein topology with a hidden Markov model: application to complete genomes. J Mol Biol. 2001;305(3):567-80.

50. Sperschneider J, Gardiner DM, Dodds PN, Tini F, Covarelli L, Singh KB, Manners JM, Taylor JM. EffectorP: predicting fungal effector proteins from secretomes using machine learning. New Phytol. 2016;210(2):743-61.

51. Emms DM, Kelly S. OrthoFinder: solving fundamental biases in whole genome comparisons dramatically improves orthogroup inference accuracy. Genome Biol. 2015;16:157.

52. Gel B, Diez-Villanueva A, Serra E, Buschbeck M, Peinado MA, Malinverni R. regioneR: an $\mathrm{R} /$ bioconductor package for the association analysis of genomic regions based on permutation tests. Bioinformatics. 2016;32(2): 289-91.

\section{Publisher's Note}

Springer Nature remains neutral with regard to jurisdictional claims in published maps and institutional affiliations.
Ready to submit your research? Choose BMC and benefit from:

- fast, convenient online submission

- thorough peer review by experienced researchers in your field

- rapid publication on acceptance

- support for research data, including large and complex data types

- gold Open Access which fosters wider collaboration and increased citations

- maximum visibility for your research: over $100 \mathrm{M}$ website views per year

At BMC, research is always in progress.

Learn more biomedcentral.com/submissions 